\title{
Spectinomycin Sulfate
}

National Cancer Institute

\section{Source}

National Cancer Institute. Spectinomycin Sulfate. NCI Thesaurus. Code C90691.

The sulfate salt form of spectinomycin, an aminocyclitol aminoglycoside antibiotic derived from Streptomyces spectabilis with bacteriostatic activity. Spectinomycin binds to the bacterial $30 \mathrm{~S}$ ribosomal subunit. As a result, this agent interferes with the initiation of protein synthesis and with proper protein elong ation. This eventually leads to bacterial cell death. 